\title{
Relaxation processes and self-trapping of excitons in rare gas solids
}

\author{
Irina Fugol \\ B.Verkin Institute for Low Temperature Physics and Engineering, National Academy \\ of Sciences of Ukraine, Kharkov, 310164, Ukraine
}

\begin{abstract}
The principal problems of electronic excitation evolution and the phenomena of self-induced lattice distortion and self-trapping of excitation in rare-gas cryocrystals are considered. The mechanisms of energy relaxation and formation of new point defects in the lattice are analyzed on the basis of spectroscopic measurements of cathodoluminescence and absorption in VUV and VIS ranges as a function of temperature and radiation dose.
\end{abstract}

\section{INTRODUCTION}

The original notion of elementary excitations in insulators goes back to the Frenkel works. Just 65 years ago Frenkel introduced a notion of exciton as an uncharged wave of excitation when considering the transformation of light into heat in monoatomic insulators, that is in rare gas solids (RGS). Later on these simplest cryocrystals served as ideal objects to study the fundamental problems of the condensed state such as the dynamics of excitons, the processes of excited state relaxation, the microscopic aspects of energy conversion from electronic excitation to atomic motions in the lattice, etc. Over the last decades we have witnessed remarkable progress in these fields of experimental and theoretical physics of cryocrystals, summarized in several reviews [1-6]. However there are still quite a number of blanks and controversile points that need further consideration. This review is primarily concerned with dynamic processes of excitons in the lattice of RGS, viz the creation of excitons, their relaxation during interaction with phonons, various self-trapping mechanisms, exciton-stimulated phenomena of defect formation, etc. The consideration is based on highly - resolved spectroscopic measurements in VUV and VIS ranges as a function of temperature, dose and energy of excitation. For short, only the experimental data for xenon and neon representing the extreme situations in the row of RGS, will be involved.

\section{ELECTRONIC STRUCTURE AND CREATION OF EXCITONS}

The rare gas cryocrystals have the widest band gap Eg, i.e. the largest excitation energy for an electronic system, and the smallest binding energy $\varepsilon_{b}$ between atoms in the lattice. Thus, the energy concentrated in electronic excitation exceeds the characteristic lattice energies almost by two or three orders of magnitude. The processes of electronic energy relaxation in RGS are of particular interest from perspective for the electronically-induced atomic rearrangement in the lattice. Dynamics of electronic and excitonic states whose spectroscopic manifestations are considered below is controlled by the band structure parameters as well as by the interaction with a phonon system. Some characteristic energies of the band structure are given in Table 1. The conduction bands derived from the excited $(n+1) s$ electrons are rather wide and experiments on drift mobilities evidence that the conduction electrons are delocalized in $\mathrm{Xe}, \mathrm{Kr}$, Ar but the question is not so clear for $\mathrm{Ne}$. The stability of the electron states in the conduction band is determined by the electron affinity $E_{A}=E_{v a c}-E_{g}$. This quantity is positive for $\mathrm{Xe}, \mathrm{Kr}$ and negative for $\mathrm{Ne}$. The latter means that exists an energy gain for quasi-solitary arrangement of electrons in the lattice of $\mathrm{Ne}$. As the electron states in the conduction band have the spherical symmetry, the exciton and hole states display anisotropic properties. According to the weak-bonding approximation the hole (valence) bands are relatively narrow and splitted by spin - orbit interaction $\left(\Delta_{\mathrm{so}}\right)$ and by interaction of atomic $\mathrm{p}$ - orbitals with crystal field ( $\Delta_{\mathrm{TL}}$ is a longitudinal - transverse splitting). The hole and exciton states are formed by interatomic overlapping integrals that depend strongly on the angle between the axis of the atomic p-state 
and the lattice vector. The lowest hole band is derived from the resonance transport of the unfilled np-state of the valence shell $n s^{2} n p^{5}$ while the corresponding lowest exciton band is derived from the transport of the Rydberg atomic excitation $n s^{2} n p^{5}(n=1) s$. The velocity of resonance transport, i.e. the energy of resonant bond of the Rydberg excitation is mainly limited by the np-hole transport. Hence, the exciton band approximately follows the hole band and differs from it only in the scaling factor reducing its width to nearly half (see Table 1).

TABLE 1. Band gaps energy, difference between atomic ionization potential and Eg, electron affinity, hole band widths, $\mathrm{n}=1(\Gamma(3 / 2))$ exciton energy, matrix shift, exciton band widths $2 \mathrm{~B}$.

\begin{tabular}{|c|c|c|c|c|c|c|c|}
\hline $\mathrm{eV}$ & $E_{g}(3 / 2)$ & $I_{\text {gas }}(3 / 2)-E_{g}(3 / 2)$ & $\mathrm{E}_{\mathrm{A}}$ & $\Delta \mathrm{E}_{\mathrm{h}}$ & $\mathrm{E}_{\mathrm{ex}}$ & $\mathrm{E}_{\mathrm{gas}}(3 / 2)-\mathrm{E}_{\mathrm{ex}}$ & $2 B$ \\
\hline $\mathrm{Xe}$ & 9.32 & 3.0 & 0.5 & 2.0 & 8.36 & 0.08 & 0.9 \\
\hline $\mathrm{Ne}$ & 21.5 & 0.05 & -1.3 & 0.5 & 17.36 & -0.69 & 0.3 \\
\hline
\end{tabular}

The exciton states are classified by the ratio between state radius $r_{n}$ and minimum interatomic spacing $d$. For the lowest excitonic state this ratio is 0.75 for $\mathrm{Xe}$ and 0.77 for $\mathrm{Ne}$ (the intermediate radius case). The optical absorption in the exciton resonance region is governed by a set of intensive peaks which are assigned to two spin-orbit split series of parabolic excitons at the $\Gamma$-point with $j=3 / 2$ and $j=1 / 2$ (Fig.1). Beyond doubt, the collection of peaks in $\mathrm{Xe}$ and $\mathrm{Kr}$ can be described by the Wannier-type series. But this procedure is not valid for $\mathrm{Ne}$. First, in Ne there is a total correlation with the Rydberg-type atomic series $2 p^{6}-2 p^{5} m s, m s^{\prime}(m>3)$ and second, the radii and binding energies calculated by the Wannier-series do not correspond to the real magnitudes. Thus, in $\mathrm{Xe}, \mathrm{Kr}$ we do observe the free band excitons in the absorption process, while in Ne the question of exciton delocalization at absorption remains open.

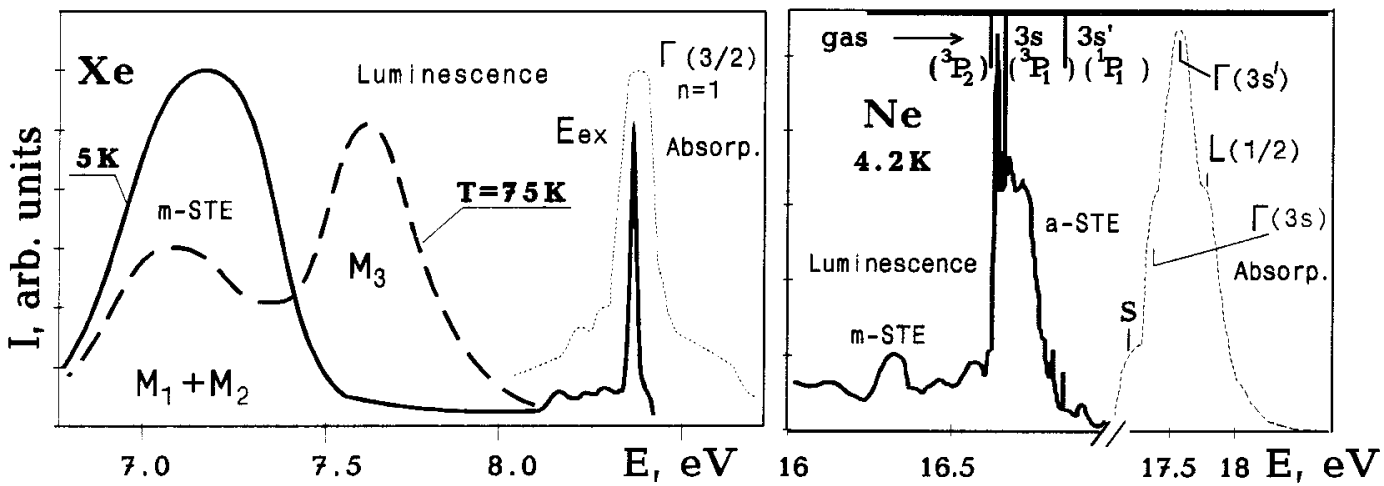

Fig. 1 Luminescence and absorption of solid xenon and solid neon.

\section{EXCITON-PHONON INTERACTION: FREE AND LOCALIZED EXCITONS SHOWN BY ABSORPTION}

The interaction of electrons and excitons with phonon fields can cause dramatic modifications in the state of band quasi-particles. In cubic isotropic crystals with a single type of acoustic phonons the energy of electron (exciton)-phonon interaction $H_{\text {int }}$ is determined by the deformation potential $\mathrm{C}$ and is mainly connected with changes of volume during deformation: $H_{\text {int }}=-\mathrm{C}$ div u (only longitudinal phonons are involved). In terms of the deformed ion model the quantity $C_{e x}$ for excitons is: $C_{e x}=C_{e}+C_{h}$, where $C_{h}$ and $\mathrm{C}_{\mathrm{e}}$ are the deformation potential of valence and conduction bands, respectively. Due to deformability of the lattice the exciton is able to induce a local distortion around itself during its life time and to be self-trapped by it. There are two characteristic energies, $D$ and $E_{L R}$ for RGS which define the energy of exciton-phonon interaction: $\mathrm{D}^{2}$-is a root-mean-square energy of exciton-phonon interaction at the initial stage of exciton creation (for absorption) and $\mathrm{E}_{\mathrm{LR}}$-is a gain in the system energy from the lattice distortion around STE (at the final stage of exciton relaxation before luminescence). In this connection two dimensionless parameters must be considered, namely: a small parameter $\lambda$ for exciton-phonon scattering and a large parameter $\Lambda$ for the self-trapping, $\Lambda \gg>1$ (see Tab.2)[2,5,7]. The basic energies and parameters of exciton-phonon interaction are listed in Tab.2. For RGS the triple inequalities are always fulfilled $h w<<D<B<E_{L R}(T a b .1,2)$. The competition between free and localized states seen by absorption is governed by a small nonadiabatic 
parameter $\lambda=\mathrm{D}^{2} / \mathrm{B}^{2}$. The competition between FE and STE seen by luminescence is governed by a large adiabatic parameter $\Lambda=E_{L R} / B$.

TABLE 2. Binding energy, average phonon energy and characteristic energies and parameters of exciton-phonon interaction for $n=1 \Gamma(3 / 2)$ in the case of one-center (a-STE) and two-center (m-STE) self-trapping.

\begin{tabular}{|c|c|c|c|c|c|c|c|}
\hline RGS $\quad$ eV & $\varepsilon_{b}$ & hø & D & $\lambda=\mathrm{D}^{2} / \mathrm{B}^{2}$ & $E_{L R}$ & $\Lambda=E_{L R} / B$ & $\mathrm{E}_{\mathrm{LR}} / \mathrm{h} \varpi$ \\
\hline $\mathrm{Xe}$ & 0.16 & 0.006 & $\leq 0.07$ & $<0.03$ & m-STE:0.85 & 1.9 & 140 \\
\hline & & & & & a-STE:0.85 & 4.2 & 130 \\
\hline $\mathrm{Ne}$ & 0.019 & 0.0075 & 0.08 & 0.3 & m-STE: 1.55 & 7.8 & 206 \\
\hline
\end{tabular}

Let us discuss the question on possibility of simultaneous occurrence of free and localized states in absorption. Generally speaking, Toyozawa et al. have pointed out, that at $E_{L R}>B$ the absorption is of a dual character [8]. But the situation with such high magnitudes of $B / h w$ and $E_{L R} / h w$ which are characteristic of RGS has been analyzed by no one. We consider now the criteria for creation of free and exciton-phonon mixing states during absorption. The condition for coherent propagation of excitons is a weak damping of the state compared to the kinetic energy of the quasi-particle

$$
\varepsilon_{k}=\left(\hbar^{2} k^{2} / 2 m_{e x}\right)>\operatorname{Im} S(E), \quad \operatorname{Im} S(E)=\Gamma(E)+G_{L}(E)
$$

where $\operatorname{ImS}(E)$ is an imaginary part of the self-energy variation due to exciton-phonon interaction $S(E)$. Damping $\Gamma(E)$ under single-phonon scattering of exciton was taken from [2], damping $G_{L}(E)$ due to localization under "multiphonon dressing" was taken from [9]

$$
\Gamma(E, T)=\frac{3}{4} \lambda T\left(\frac{2 E}{m_{e x} s^{2}}\right)^{1 / 2} \cdot\left[1+\frac{m_{e x} s^{2}}{2 E}+\ldots\right] \quad G_{L}(E)=\sqrt{2 \pi} \frac{\left(E-E_{M}\right)^{2}}{D} \exp \left[-\frac{\left(E-E_{M}\right)^{2}}{2 D^{2}}\right]
$$

Numerical calculations of exciton damping in comparison with the kinetic energy of excitons are shown in Fig. 2 for $\mathrm{Xe}$ and for $\mathrm{Ne}$. It is seen that for $\mathrm{Xe}$ there is a region below the dotted curve which represents free excitons with $\varepsilon_{k}>\Gamma_{k}(E)$ and $\varepsilon_{k}>G_{l}(E)$. For Ne over the whole energy region of excitonic resonance the inequalities $G_{L}(E)>\varepsilon_{k}>\Gamma_{k}(E)$ are valid. The effect of exciton-phonon coupling on absorption spectra has been estimated in two limiting approaches: weak exciton damping [9] and strong exciton- phonon coupling [8]. For Xe separated bands of almost free and localized states were obtained (the integral intensity of the latter is about $3 \%$ ). In Ne the only absorption band is presented under nonadiabatic mixing of free and vibronically excited localized states [10].
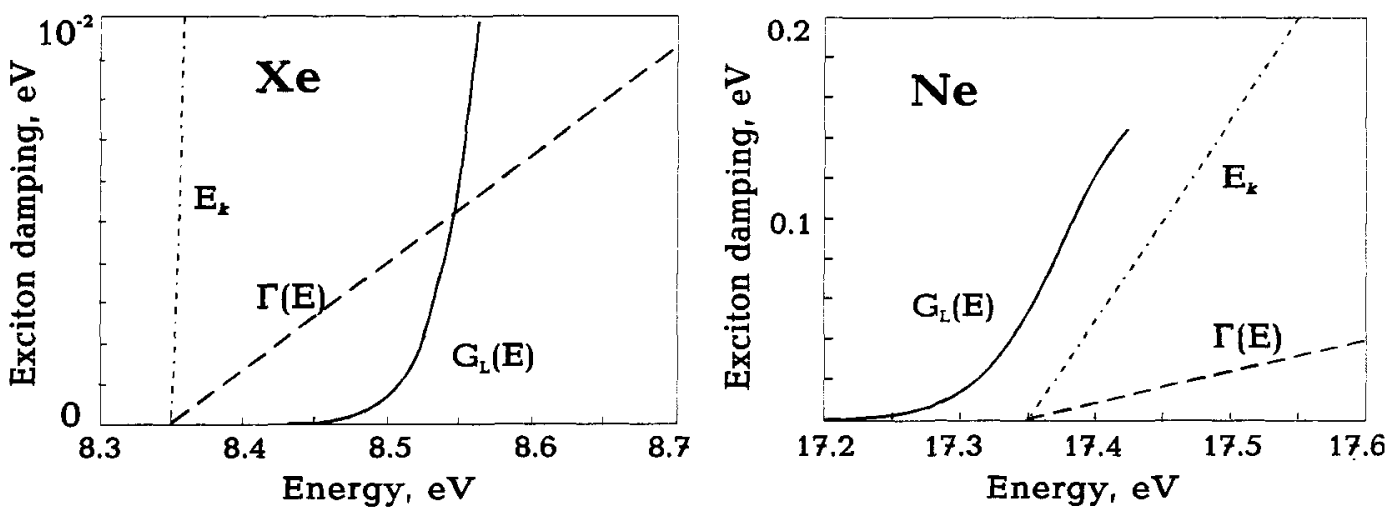

Fig. 2 Kinetic energy and damping of excitons for solid xenon and solid neon.

\section{DIFFERENT CHANNELS OF CREATION AND POPULATION OF STES. COEXISTENCE OF FE AND STE IN LUMINESCENCE}

As we have shown, the excitons are in free or/and unrelaxed (nonadiabatic) localized states immediately after optical creation. During exciton radiation lifetime $\tau_{\text {rad }}$ the possibility exists of developing the relaxation process with strong lattice distortion which result in a energy gain of a composite system 
consisting of a local lattice deformation and a particle trapped by it: $\mathrm{E}_{\mathrm{LR}}>\mathrm{B}, E_{L R}=C_{e x}^{2} / 2 M s^{2}$. The transition from FE to STE goes on with overcoming a barrier to ST-ing. According to [7] at $\mathrm{T}<\mathrm{h} \varpi / \Lambda$, the $\mathrm{ST}$ rate is given by the expression

$$
\Gamma_{S T}(T)=\pi(B / \pi)^{3 / 2} \exp \left(-x B / \Lambda^{2} \varpi\right)
$$

$x B / \Lambda^{2}$ being the height of the ST barrier. In the RGS lattice there are a variety of local relaxed configurations which lower the common energy of the system around STE. One of the main factors defining the channel of the lattice relaxation is a competition between the contributions of hole $\left(C_{h}\right)$ and electron $\left(\mathrm{C}_{\mathrm{e}}\right)$ deformational potentials to $\mathrm{C}_{\mathrm{ex}}$. It should be particularly emphasized that the contribution of $\mathrm{C}_{\mathrm{h}}$ is strongly dependent on the angle between polarization wave vector $\mathbf{k}$ and lattice vector $\mathbf{l}$. As is shown in [11], the overlapping integrals for transverse $\left(\mathrm{J}_{\mathrm{I}}\right)$ and longitudinal $\left(\mathrm{J}_{\mathrm{I}}\right)$ holes (excitons) are distinguished by a factor of eight $\left(J_{\Pi}>J_{I}\right)$. Thus, the contribution of $C_{h}$, to $C_{e x}$ reduces remarkably for transverse excitons. In the Xe lattice of high polarisability an anisotropic attraction for all angles of the excited atom interaction is predominant, i.e. $C_{e x} \simeq C_{h}\left(\left|C_{e}\right|<C_{h}\right)$. In the Ne lattice the $C_{h}$ contribution is four times less than for Xe and at least for transverse excitons is predominant an isotropic repulsion $\left(C_{e}\right)$, i.e. $C_{e x}=C_{e}\left(\left|C_{e}\right|>C_{h}\right)$. In Xe only a two-center channel of $\mathrm{m}$-ST-ing with a redistribution of the resonant bonds to two atoms (excimer) is realized (Fig.1). In Ne a one-center channel of ST-ing with a creation of a bubble and a break of the resonant binding around a-STE is more favorable (Fig.1). The cooperation of the defect (impurity) potential with the exciton-phonon coupling enhances the ST efficiency. In this case the defect (impurity)assisted extrinsic ST-ing with an efficient lowering of the ST-barrier takes place. There is another possibility of direct population of STE states via hole-electron recombination. The m-STE states are populated under radiative recombination ( $\mathrm{m}-\mathrm{STh}$ with free or trapped electrons). The a-STE states are populated under dissociative recombination ( $\mathrm{m}$-STh with free or trapped electrons). When stating the concept of coexistence of FE and STE the population of STE via e-h recombination as well as through extrinsic ST-ing was not involved. This conception concerns only intrinsic STE. The effect of coexistence is supported by the sophisticated treatment of luminescence in $\mathrm{Xe}, \mathrm{Kr}$ and $\mathrm{Ar}$, where the resonant peaks and bands with the Stokes shift are observed simultaneously [2,3]. Evidence of the FE and $\mathrm{m}$-STE coexistence is rather convincing for Xe (Fig. 1): i) there exists a $\mathrm{M}_{2}$ component in $\mathrm{m}$-STE which is independent of dose irradiation, i.e. it is avaliable in the regular lattice [12]; ii) the time resolved spectroscopy confirms the view that the FE-peak is a precursor of the state emitting a fast component of the $\mathrm{M}_{2}$-band [13]; iii) the intensity of the FE-peak is independent of temperature up to $50 \mathrm{~K}$, in agreement with tunneling through the STbarrier (Fig. 3a) $[13,14]$; iv) the value of $\tau_{S \mathrm{r}}=2.10^{-8} \mathrm{~s}$ measured in [13] satisfies the condition for occurrence of FE in luminescence: $\tau_{\mathrm{rad}}=2 \cdot 10^{-9} \mathrm{~s}<\tau_{\mathrm{ST}}$.
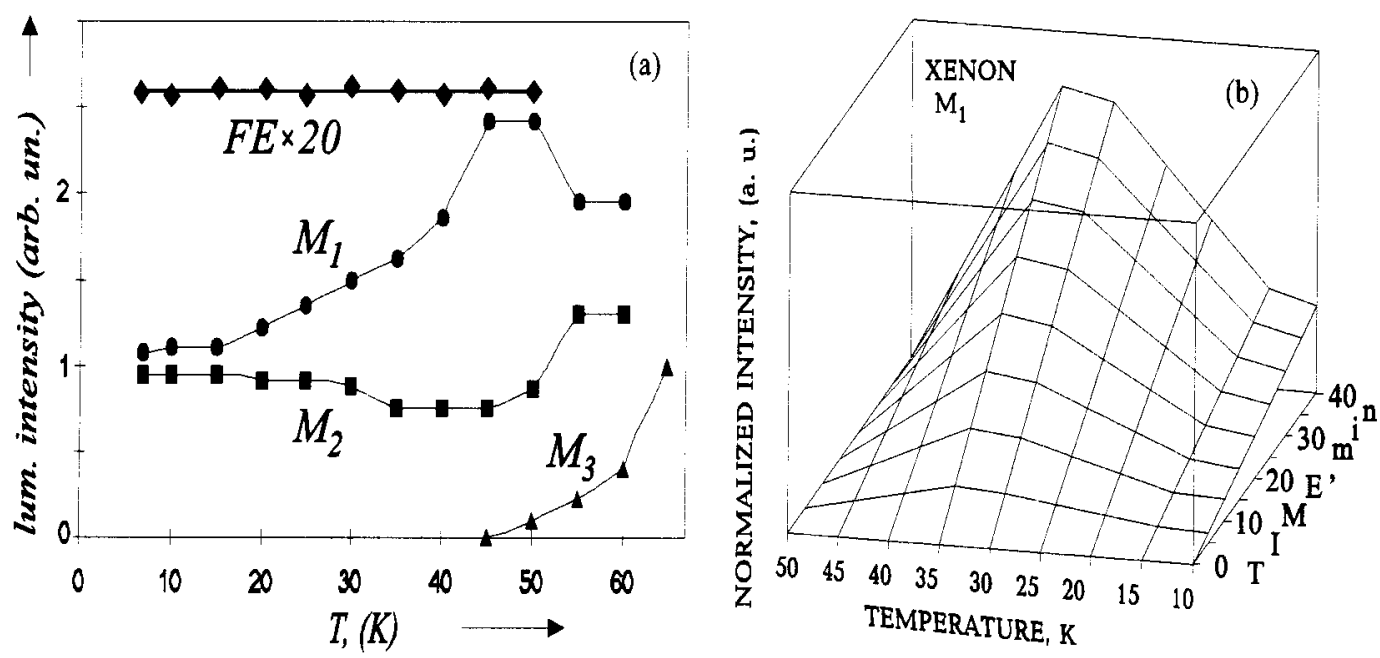

Fig. 3 Temperature dependencies of cathodoluminescence intensities of FE and m-STE (a); and dose dependencies of $\mathrm{M}_{1}$-STE at different temperatures (b). 


\section{EXCITON-INDUCED DEFECT FORMATION UNDER m-STE-ing}

The luminescence spectroscopy of high resolution evidences that the multicomponent structure is observed for each band of the bulk m-STE and a-STE centers in RGS and only the intensity of one of the components $\left(\mathrm{M}_{2}\right.$ for $\left.\mathrm{m}-\mathrm{STE}\right)$ is independent of irradiation at small doses and decreases at elevated doses. Other bulk components are intensified under irradiation with time, suggesting accumulation of stable defects in RGS. The defects intercept FE energy resulting in an increase of the bulk ST-ing at the expense of the surface ST-ing. Anticorrelation of the $M_{1}$ and $M_{2}$ behaviors is also displayed by the temperature dependence above $15 \mathrm{~K}$ (Fig. 3a). From the general point of view the ST process can be considered as a loss of the lattice stability induced by the electronic subsystem and abrupt changes of the local topology of the adiabatic surface. Such a fast atomic rearrangement around STE can be topologically described by using the method of the mathematical theory of elementary catastrophes in the bifurcational plurality [15]. In this sense the STE is a transient "defect" state and the process of permanent defect creation becomes possible under the condition $\Delta \mathrm{E}>>\varepsilon_{\mathrm{b}}$ and $\tau_{\mathrm{ST}}>\tau_{\mathrm{df}}$. The first inequality means that the energy $\Delta \mathrm{E}$ released on ST-ing exceeds the binding energy $\varepsilon_{\mathrm{b}}$, and the second inequality requires that defects should be formed and stabilized during the ST-time (i.e. $\tau_{\mathrm{df}} \leq 10^{-8} \mathrm{~s}$ for Xe). Nowadays it is well known that the m-STE decay provokes a creation of stable point defects (Frenkel pairs) at the off-center position and a reorientation of the excimer molecule in alkalihalides [6]. In [12] it is shown that DF under m-STE in Xe occurs not only at the final stage of m-STE but also in the course of energy relaxation during the lifetime of excited states (for long-living states). In Fig. $3 b$ the dose dependencies of the m-STE intensity at different temperatures are shown for $\mathrm{M}_{1}$ component in $\mathrm{Xe}$. The slopes of the dose dependencies $\mathrm{dI} / \mathrm{dt}$ are a nonmonotonic function of temperature with a maximum at $35 \mathrm{~K}$. Above $\mathrm{T}>50 \mathrm{~K}$ there is no temperature effect. The maximum in the DF-efficiency and its decrease at higher temperature implies that the DF-mechanism in the $4-60 \mathrm{~K}$ range is not consistent with the motion of the initial vacancies presented before irradiation. Indeed, the diffusion coefficient of external vacancies at $\mathrm{T}<60 \mathrm{~K}$ is small $\mathrm{D}_{\mathrm{v}}<10^{-26} \mathrm{~cm} . \mathrm{s}^{-1}$ and has no maximum vs $\mathrm{T}$. Thus, the defects are formed during $\mathrm{m}$-ST-ing but at which stage of it? The DF-channel at the final stage of the $\mathrm{m}$-STE decay due to the transition to a repulsive curve with $\Delta \mathrm{E}_{0} \cong 0.5 \mathrm{eV}$ is an athermic channel and manifests itself purely in the $5-15 \mathrm{~K}$ temperature range when the correlation of $\mathrm{M}_{1}$, and $\mathrm{M}_{2}$ intensities is observed (Fig. 3a). The DF-channel at the first stage of m-ST-ing (in the excited state) is a temperature dependent channel for molecular states ${ }^{3} \Sigma_{u}^{+}$of Xe the lifetime $\tau_{R}(T)$ of which decreases with increasing temperature. The number of permanent defects originated per unit time via the "excited state" channel is determined as follows:

$$
d N_{d f}^{*} / d t \propto \Gamma_{S T} \cdot \tau_{d f}^{-1}(T) \cdot N\left(\tau_{R}(T), \tau_{d f}(T)\right), \text { and } d N_{d f}^{*} / d t \propto d I(T) / d t
$$

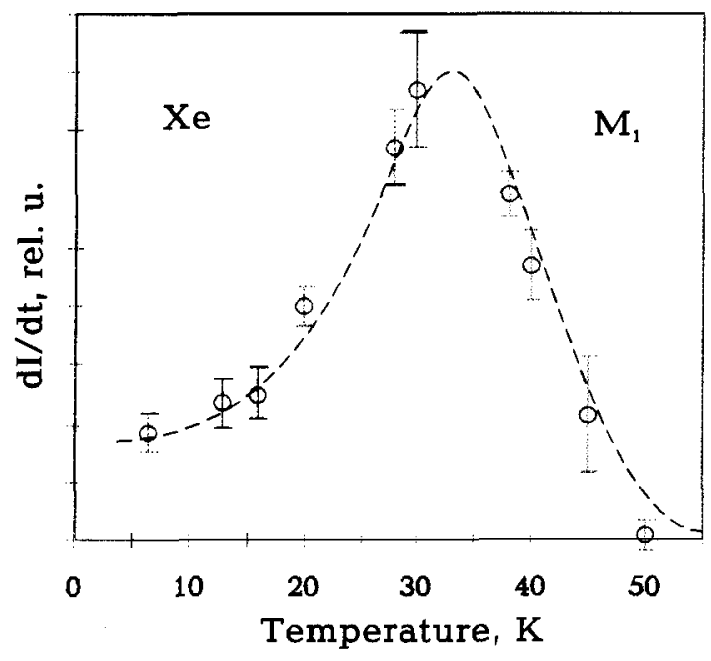
Fig. 4 Temperature dependencies of defect
Here $N\left(\tau_{R}(T), \tau_{d f}(T)\right) \propto \exp \left(-\tau_{d f}(T) / \tau_{R}(T)-\right.$ characterizes the change in ${ }^{3} \Sigma_{u}^{+}$population in a time $\tau_{d f} ; \tau_{d f}^{-1} \propto \exp \left(-E_{a}^{d f} / k T\right)$ in assumption of a thermo - activated process of DF. In Fig. 4 the dashed line shows the results calculated by (5). The calculated curve describes well the experimental dependencies of the defect accumulation rate $\mathrm{dI}_{\mathrm{M} 1} / \mathrm{dt}$ for the following values of parameters: $\tau_{\mathrm{df}}=4.2 \cdot 10^{-7} \mathrm{~s}$, $E_{a}^{d f} \cong 1.5 \cdot 10^{-2} \mathrm{eV}$, for $\tau_{\mathrm{R}}(\mathrm{T})$ we used the data on time for ${ }^{3} \Sigma_{u}^{+}$state given in [3]. The analysis made favors the view that the possibility of the permanent point defect formation exists at all stages of the excitonic STrelaxation to molecular centers. The $M_{1}$ band of m-STE for the stable defect position (off-center excimer) is shifted to a red region from the $\mathrm{M}_{2}-\mathrm{STE}$ band for the reversible STE-configuration (on center excimer) in energy $\mathrm{E}\left(\mathrm{M}_{2}\right)-\mathrm{E}\left(\mathrm{M}_{1}\right) \cong 0.2 \mathrm{eV}$. 


\section{EXCITON-INDUCED DEFECT FORMATION UNDER a-ST-ing}

The main contribution to the luminescence of solid neon in the VUV region is made by a-STEs that occur in the vicinity of the gaseous transitions $\left({ }^{3} \mathrm{P}_{2},{ }^{3} \mathrm{P}_{1},{ }^{1} \mathrm{P}_{1}\right)$ with a small matrix shift relative to that of gas and a large Stokes one in relation to unrelaxed excitons in absorption (Figs. 1 and 5). The a-STE distinguishing feature is a rich fine structure consisting of three bulk (" 2 ", " 1 ", " $d$ ") and two surface ("0", " $s$ ") components for each of the atomic states [2,3]. It should be mentioned that many of the impurity centers in $\mathrm{Ne}$ also exhibit a structure similar to that of the bulk components of a-STE of pure neon [16] (Fig. 5). Taking into account the fact that in Ne predominant are repulsion forces, the a-STE microscopic structure can be adequately given in terms of the model "an excited atom in a bubble".

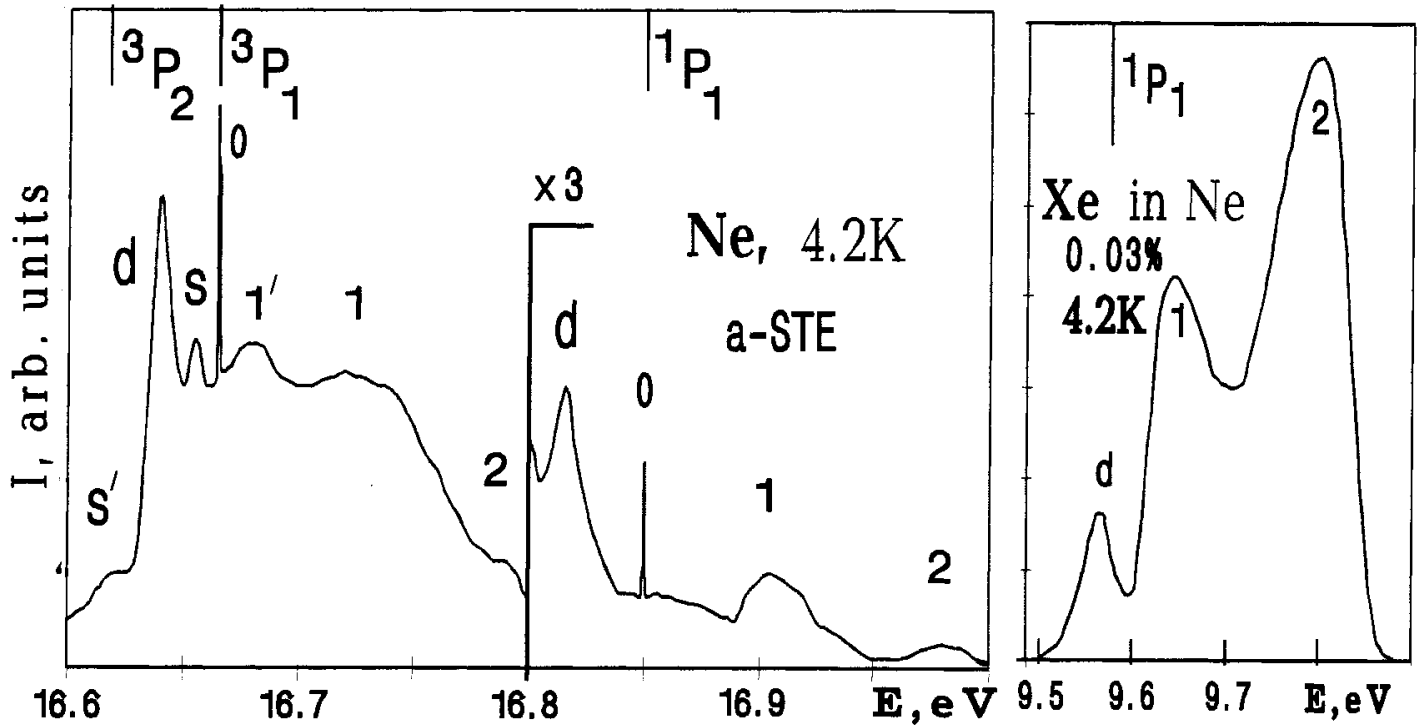

Fig. 5 Multicomponent structure of a-STE bands of luminescence in pure Ne and Xe in Ne.

When being under the action of negative isotropic deformation potential, $-C_{e x} \sim-C_{e}$, the exciton tends to push apart the surrounding lattice so that the energy gain $\mathrm{E}_{\mathrm{LR}}$ goes mainly to radial atomic displacements, $r$. In parallel with the first rough estimations of bubble dimensions under the assumption "ut tensio sic vis", [2] a great body of comprehensive calculations within different approximations was made [6]. The estimates of

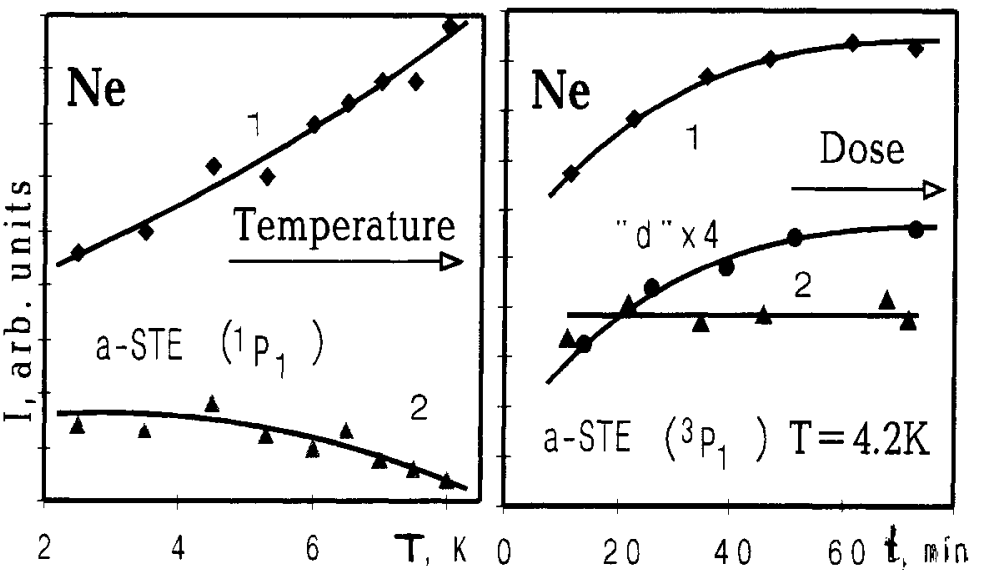

Fig. 6 Temperature (a) and dose (b) dependencies of the a-STE intensity for "1","2" and "d" components in solid $\mathrm{Ne}$ atomic displacement in the first coordination sphere, $\Delta \mathrm{r}_{1} \cong$ $(0.2-0.3) r_{1}$, and bubble radius, $\mathrm{r}_{\mathrm{b}} \sim 3.8-4.1 \AA$, obtained by different approximations are in not so poor agreement with each other. Note that the deformation involves more than ten coordination spheres. The calculated matrix shifts under a-STE for a symmetric displacement prove to be consistent with the position of component " 2 ". The temperature variations in intensity of the bulk components (Fig. 6a) and their dose dependencies (Fig. 6b) suggest that component " 2 " should be indeed ascribed to the relaxation of the regular lattice with a reversible distortion. At the same time components " 1 " and " $\mathrm{d}$ " may be attributed to different metastable stages of a more profound relaxation with a local distortion of the cubic symmetry. As the dose is increased, the intensity of components " 1 " and (Fig. 6a and 6b) " $\mathrm{d}$ " becomes 
higher, i.e. there occur a generation and an accumulation of bulk defects under a-STE. The possibility of developing a cavity with the symmetry breaking by the pattern of microplastic deformation is considered in [11]. According to [11], if the radius of the first coordination sphere increases by a factor of $\mathrm{w}\left(\mathrm{r}+\Delta \mathrm{r}_{1}\right)=\mathrm{wr}_{1}$ and the sphere surface by a factor of $\mathrm{w}^{2}$, the extended sphere accommodates $12 \mathrm{w}^{2}$ atoms instead of initial 12, i.e. $\Delta \mathrm{N}=12\left(\mathrm{w}^{2}-1\right)$ extra: for $\mathrm{Ne}^{*} / \mathrm{Ne} w=1.22$, i.e. $\Delta \mathrm{N} \cong 5.8$, for $\mathrm{Xe} / \mathrm{Ne} w=1.4$, i.e. $\Delta \mathrm{N} \cong 11.5$. For $\mathrm{N} \gg>1$ a rearrangement of the surrounding lattice with a more dense population of the first coordination sphere and an increase in the number of vacancies inside the sphere, i.e. a generation of point defects of a vacancy-interstitial atom type, is more favorable as regards minimum energy consumption. If the average energy of the formation of a vacancy near a-STE in Ne is assumed to be $h \sim 0.015 \mathrm{eV}[5,6]\left(h<h_{0}=0.02 \mathrm{eV}\right.$ for the vacancy formation in the regular lattice), the displacement $E_{21}$ from configuration "2" to configuration " 1 " is associated with a further extension of the cavity for 4-5 vacancies for pure $\mathrm{Ne}$ $\left(E_{21}=0.065 \mathrm{eV}\right)$ and for 9-10 vacancies for $\mathrm{Xe}$ in $\mathrm{Ne}\left(E_{21}=0.14 \mathrm{eV}\right)$, in good agreement with the possibility of additional population of the sphere under DF [11]. It should be noted that the narrow component " $d$ " corresponds to a slight red shift relative to the gas, that is typical for predominance of polarization attraction for bubble dimensions higher than the equilibrium ones. The situation is realized if the a-ST-ing occurs in the vicinity of a cluster of vacancies accumulated due to the exciton-induced DF under a-STE. The deciding factor for relaxation with stable defect formation is a condition $\tau_{\mathrm{ST}}>\tau_{\mathrm{df}}$. According to [2], the ratio of intensities of components " 2 " and " 1 " for different impurity centers in solid Ne correlates well with the time of population of these centers from higher-lying states. Another evidence for the significance of the time condition may be the data given in [17] for selective excitation of a-STE: component " 1 " is absent on a-STE excitation to the lowest band $\Gamma(3 \mathrm{~s})$ and observed only on excitation from higher exciton bands. It should be mentioned that some authors have attempted to treat the manifold structure of a-STE in Ne in terms of the motion of external (external) vacancies $[5,17]$. But we suppose that this mechanism is valid only at elevated temperatures ( $\mathrm{T}>19 \mathrm{~K}$ for $\mathrm{Ne}$ ). Another interesting possibility of investigating the a-STE process in solid Ne exists when observing "hot" luminescence in the visible spectrum region of 1.65-2.1 eV [18]. In this region there occur transitions from higher 3p: states corresponding to the excitons of the Brillouin zone $\mathrm{X}$-point to $3 \mathrm{~s}_{\mathrm{k}}$ states. Of the VIS luminescence typical also is the multicomponent structure, but channel " 1 " corresponding to the nonsymmetric atomic displacement and the induced DF is invariably a dominant channel of the bulk a-STE. It is natural that the enhancement of component "1" in the VISspectrum would be ascribed to the anisotropy of $-\mathrm{C}_{\mathrm{e}}$ due to that of the electron cloud for an outer-shell electron of $\mathrm{p}_{\mathrm{i}}$-states

\section{EVIDENCE OF ELECTRON ST-ing IN Ne}

As is known, the recombination of holes and free electrons in heavy RGSs makes the main contribution to the population of free excitons [4]. Consideration of the situation in Ne suggests that there exists an additional channel of dissociative recombination with a direct population of STE states $\left[\mathrm{Ne}_{2}{ }^{+}+\mathrm{e}^{-}\right]$[20]. In the context of recombination population of a-STE in Ne with the participation of STe, we can show the results on T-dependence of the total intensity of a-STE cathodo-luminescence and T-variations in the ratio of bulk and surface luminescences for VUV and VIS bands (Fig.7). It is evident that as T is increased: (i) the total luminescence intensity, $I_{\text {tot }}$, increases; and (ii) the ratio $I_{\text {bulk }} / I_{\text {surf. }}$ reduces for the VUV bands and (iii) that increases for the VIS ones. The above data can be reasonably treated with taking into account the

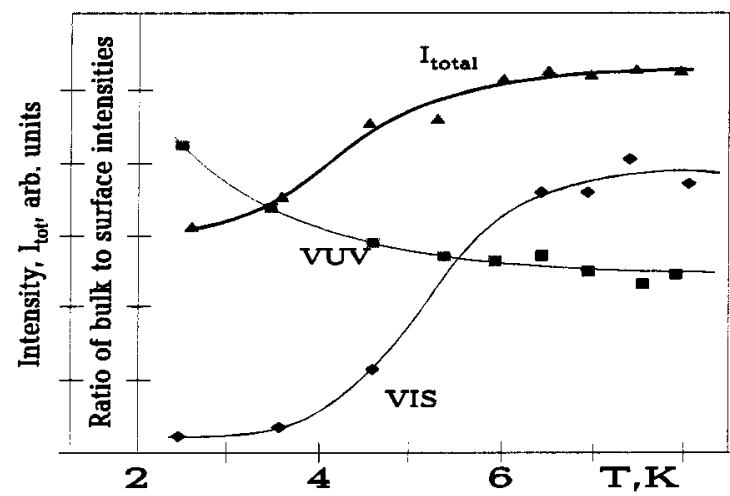

Fig. 7 Total intensity $I_{\text {tot }}$ and ratio $I_{\text {bulk }} / I_{\text {surf }}$ for VUV and VIS as function of $T$.

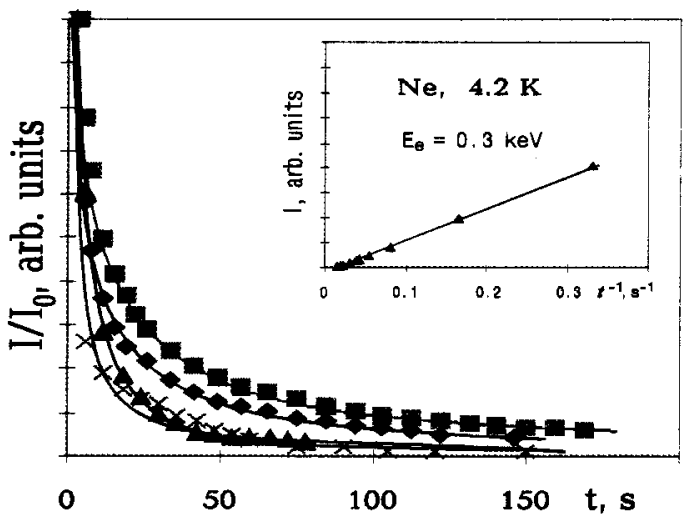

Fig. 8 Afterglow of pure neon $(x, \Delta)$ and impurity centers in Ne: Oxygen $(\triangleleft \triangleright), \mathrm{Xe}(\square)$. 
fact that the hopping mobility of self-trapped electrons (of a small depth of the well) and localized excitons enhances with increasing $T$. The former promotes a more efficient population of the bulk luminescence centers through the recombination involving STe and the latter contributes to a faster bulk - to surface energy transport by localized excitons. The opposite T-dependencies for VUV and VIS bands suggest that the lower $\left\{3 \mathrm{~s}_{\mathrm{k}}\right\}$ states are mainly populated through the localized states of excitons in their subsequent relaxation to a-STE while the $\left\{3 \mathrm{p}_{\mathrm{i}}\right\}$ - states are principally populated due to the recombination of holes with self-trapped electrons.

One further unique property of the luminescence of $\mathrm{Ne}$ is the existence of intense and prolonged afterglow for VUV and VIS bands. In Fig. 8 shown are the afterglow curves for pure $\mathrm{Ne}$ and impurity centers oxygen $\left({ }^{5} \mathrm{~s}-{ }^{3} \mathrm{P}\right) 0^{*}$ and xenon $\left({ }^{3} \mathrm{P}-{ }^{1} \mathrm{~S}_{0}\right) \mathrm{Xe}$. It should be mentioned that all the luminescence bands shown have their own radiation lifetimes of approximately a few tens of ns, i.e. the prolongs afterglow is direct testimony to recombination delay. The characteristic times of the afterglow are of several seconds though the afterglow is observed over hundreds of seconds. The afterglow kinetics is described by a simple hyperbolic relation $I(t)=I_{0}(1+t / \tau)^{-1}$, corresponding to a two-particle recombination process (see the inset in Fig.8). The rough estimates of characteristic decay times, $\tau=\left(n_{e}{ }^{0} v \sigma\right)^{-1}$ give $n_{e}^{0} \sim 10^{13} \mathrm{~cm}^{-3}$ for the number of electrons involved in the recombination, i.e. of the same order of magnitude as the number of proper electrons generated on cathodo-excitation [18].

\section{CONCLUSIONS}

It seems that the recent surge of interest in the basics physics of RGS excited states has resulted in advances that deepen our understanding of same aspects such as the coexistence of free and localized excitonic states, the mechanisms of ST-ing and the following exciton-induced processes of atomic rearrangements in the lattice due to conversion of electronic energy to atomic degree of freedom. The creation and the temporal evolution of excitons in the lattice are controlled by two contraposed tendencies of the matter - the continual and discrete ones.

For clarity I have not touched on some problems with inherent contradictions. But such problems do exist, especially in the field of exciton kinetics and energy transfer. Despite their apparent simplicity, the wide-gap cryocrystals display an intriguing range of unresolved complexities which are still fascinating for further research.

\section{REFERENCES}

1. J.Jortner: in Vacuum Ultraviolet Radiation Physics (E.E.Koch, R.Haensel, C.Kunz, ed.) p.263. Vieweg- Pergamon, Braunschweig, (1974).

2. I.Ya.Fugol' Adv. Phys. 27, 1 (1978); 37, 1 (1988)

3. G.Zimmerer: in Excited-State Spectrroscopy in Solids, p.37, XCVI Corso Societa Italiana di Fisica,Bologna (1987)

4. N.Schwentner, E.E.Koch, J.Jortner: Electronic Excitation in Condensed Rare Gases, Springer Tracts Mod. Phys. 107 (1985).

5. M.Ueta, H.Kanzaki, K.Kobayashi, Y.Toyozawa E.Hanamura: in Excitonic Processes in Solids, Springer Series in Solid State Sciences, 60 (1985).

6. K.S.Song, R.T.Williams: Self-Trapped Excitons, Springer-Series in Sol. St. Sc. 105 (1993).

7. A.S.Ioselevich, E.I.Rashba: in Quantum Tunneling in Condenced Media, (Yu.Kagan, A.J.Leggett, ed) Elsevier Science Pub., (1992).

8. K.Cho, Y.Toyozawa J. Phys. Soc. Japan 30, 1555 (1971).

9. A.Sumi J. Phys. Soc. Japan 47, 1538 (1979).

10. I.Ya.Fugol, E.I.Tarasova Low Temp. Phys. (in press).

11. A.M.Ratner, I.Ya.Fugol' Low Temp. Phys. 13, 90 (1987), 19(3), 227 (1993).

12. I.Ya.Fugol', A.N.Ogurtsov, et. al. Low Temp. Phys. 18(1) 2731 (1992).

13. D.Varding, I.Reimand, G.Zimmerer phys. stat. sol. (b), 185, 301 (1994).

14. I.Ya.Fugol', E.N.Savchenko: in Cryocrystals (B.Verkin,A.Pricht'ko ed.), pp.360-523 Naukova Dumka, Kiev (1983).

15. V.I.Zakharov, L.N.Shchur Sov. Phys. -J.E.T.P. 54(81), 2019 (1981).

16. A.G.Belov, I.Ya.Fugol', E.M.Yurtaeva Sov. J. Low Temp. Phys. 18(2), 123 (1992).

17. W.Laasch, H.Hagedorn, T.Kloiber, G.Zimmerer phys. stat. sol. (b) 158, 753 (1990).

18. A.G.Belov, 'G.Gorbulin, E.Yurtaeva, Ya.Fugol' Low Temp. Phys. (in press). 